\title{
Binary Segmentation Masks for Registration of Bone Structures in CT Images
}

\author{
Oleg Museyko ${ }^{1}$, Fabian Eisa ${ }^{1}$, Andreas Hess ${ }^{2}$, Peter Zerfass ${ }^{1}$, Willi Kalender ${ }^{1}$, \\ Klaus Engelke ${ }^{1}$ \\ ${ }^{1}$ Institut für Medizinische Physik, Friedrich-Alexander Universität Erlangen-Nürnberg \\ ${ }^{2}$ Institut für Pharmakologie und Toxikologie, Universität Erlangen-Nürnberg \\ oleg.museyko@imp.uni-erlangen.de
}

\begin{abstract}
Rigid registration of bone structures in stacks of CT images can be improved by using a binary segmentation mask for the registration compared to using the segmented grey values directly. Three criteria are applied to the different reference and template datasets in order to quantify registration results. For all three criteria a statistically significant superiority of the technique using the binary segmentation masks is demonstrated. First results for a multimodal registration of a $\mu \mathrm{CT}$ with $\mu \mathrm{MR}$ dataset also show better results if binary segmentation masks are used for the registration.
\end{abstract}

\section{Introduction}

The accurate rigid registration of serial 3D CT datasets of the spine and the hip to determine for example longitudinal changes of geometry or bone mineral density requires an initial segmentation to separate individual vertebrae or the femur from the acetabulum. Otherwise the different relative position of multiple bones in baseline and follow-up image stacks will result in inadequate registration results. In this contribution we investigated whether the registration result can be improved by using a binarized segmentation mask instead of the segmented grey value structures. This is an important question, in particular in the presence of high noise levels as the segmentation may be less affected by noise than the registration process. Also the registration of multimodal datasets may benefit from the use of binarized segmentation masks. While for bone various registration $[1,2,3]$ and segmentation $[4,5]$ methods exist, to our knowledge this problem has never been investigated.

\section{Materials and methods}

We used the 3D rigid intensity based registration method implemented in ITK. Specifically, for all registrations a gradient flow algorithm was used to find the (local) extremum of a registration energy function, sum of squared differences (SSD) for monomodal registration and Mattes mutual information (MMI) for multimodal registration. The multiresolution $3 \mathrm{D}$ versor-based rigid body registration was applied. 


\subsection{Spine and femur CT datasets}

Pairs of CT datasets from the proximal femur and the lumbar spine from 30 human subjects each were acquired at baseline (reference) and after 12 months (template). All CT datasets were segmented by a combination of global and adaptive local thresholds followed by morphological operations as reported by Kang et al. [4] for the femur and by Mastmeyer for the vertebrae [5]. For example, Fig. 1 shows two orthogonal multiplanar reformations (MPRs) of a segmented femur. Fig. 2 shows the segmentation result in an axial MPR along with a binarized mask. Registration was performed using grey value segmentations (reg1) and binary masks (reg2).

Figure 3 shows axial MPRs of the 3D difference datasets (template minus reference) for the two registrations (reg1 and reg2). Three criteria were used to quantify the registration accuracy: the normalized (i.e. divided by the volume of the union) symmetric difference of binary reference and template mask (XOR), which equals the relative number of voxels that are not in the intersection of the two datasets; the values of the registration criterion itself (mean sum of squared differences, SSD); and the mean sum of absolute differences (SAD), computed in the union of corresponding template and reference binary datasets.

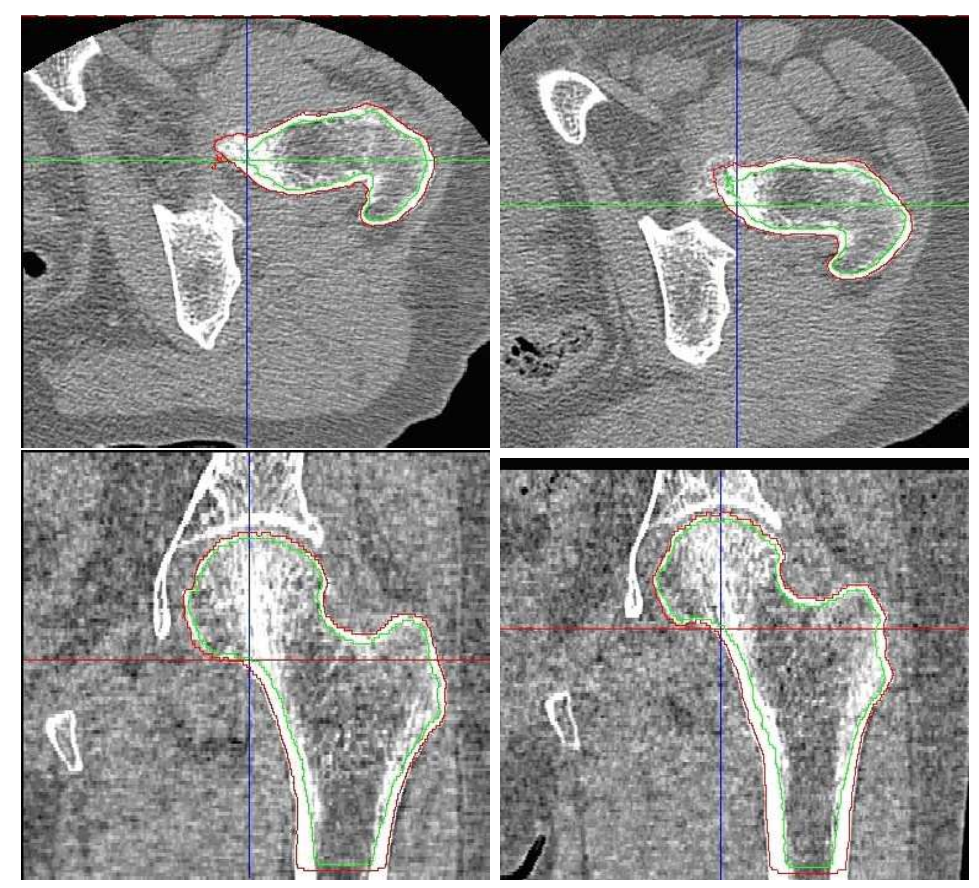

Fig. 1. Baseline (left) and follow-up (right): MPRs of the femur with periosteal and endosteal segmentation results. Note that patient positioning slightly differed between the two scans. 
Table 1. Femur and spine: difference $\Delta$ between reg1 and reg2 for SSD, SAD and $\mathrm{XOR}(\%)$ (non-parametric Wilcoxon test, number of experiments 30).

\begin{tabular}{lcccccc}
\hline & Femur & & \multicolumn{3}{c}{ Spine } \\
Criterion & $\Delta \mathrm{SSD}$ & $\Delta \mathrm{SAD}$ & $\Delta \mathrm{XOR}(\%)$ & $\Delta \mathrm{SSD}$ & $\Delta \mathrm{SAD}$ & $\Delta \mathrm{XOR}(\%)$ \\
\hline Positive ranks sum & 445 & 447 & 275.5 & 389 & 329.5 & 256 \\
Negative ranks sum & -20 & -18 & -24.5 & -76 & -105.5 & -69 \\
p-value & $3.5 \cdot 10^{-7}$ & $2.4 \cdot 10^{-7}$ & $5 \cdot 10^{-5}$ & 0.0004 & 0.007 & 0.0053 \\
\hline
\end{tabular}

\section{$2.2 \mu \mathrm{CT}$ and $\mu \mathrm{MR}$ datasets}

Figure 4 demonstrates reg1 and reg2 for multimodal 3D registration of a $\mu \mathrm{MR}$ $(78 \times 78 \times 156 \mu \mathrm{m})$ and a $\mu \mathrm{CT}(40 \mu \mathrm{m}$ isotropic $)$ dataset from a rat knee.

\section{Results}

For each of the 30 spine and hip datasets the two registrations, reg1 and reg2, were compared by first computing each quality criterion separately in the two image stacks resulting from reg1 and reg2. Then the difference $\Delta$ for each quality criterion was determined for all 30 datasets. Table 1 summarizes the results for $\triangle \mathrm{SSD}, \triangle \mathrm{SAD}$, and $\triangle \mathrm{XOR}(\%)$ for the proximal femur and the spine. The significance of the difference of the two registrations reg1 and reg2 was verified using a one-side Wilcoxon test, which is a non-parametric test (parametric tests are not suitable for these quality values).

In all cases the registration based on the binary masks lead to better results: p-values $<0.05$ indicate significant differences. The advantage of binary masks for registration holds also for the multimodal registration $(\mu \mathrm{MR}$ to $\mu \mathrm{CT})$ for the example in Fig. 4, where the growth plate and the trabecular structure indicate better registration results with binary masks.

\section{Discussion}

We have demonstrated that for clinical CT datasets the registration of binary segmentation masks is superior to the registration of grey value based segmentations. Of course this procedure requires an adequate segmentation technique.
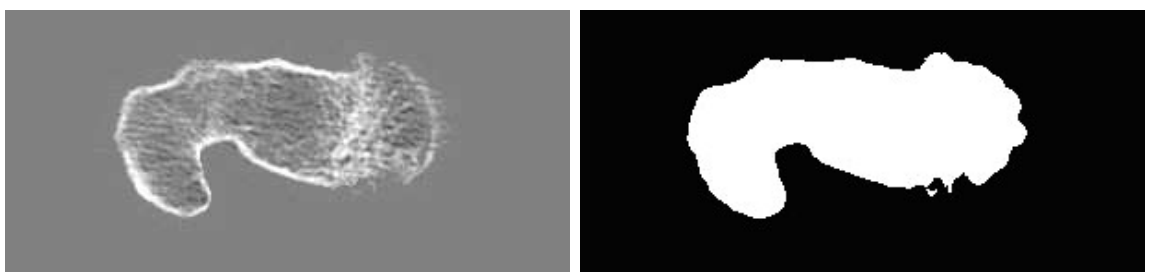

Fig. 2. Left: grey value segmentation results; right: binary segmentation mask 
This effect is also very important for small animal imaging in cases such as $\mu \mathrm{CT}$ - $\mu \mathrm{MR}$ registration where the spatial resolution of the two datasets may be different. Our results still have to be statistically confirmed for this multimodal application but we have shown the statistical significance for CT-CT applications of the spine and the proximal femur. The comparison of the values of the registration criterion for the two registrations shows that the registration of grey value images converges to a local minimum, which is different from the more optimal solution obtained with reg2. This suggests that the structural differences in a pair of images are strong enough to deteriorate the registration, but do not have that influence on the segmentation. Thus, the binarization of the bone images improves the efficiency of the registration optimization algorithm. We have also shown that the results were largely independent of the quantitative criteria used for the measurement of the registration quality.

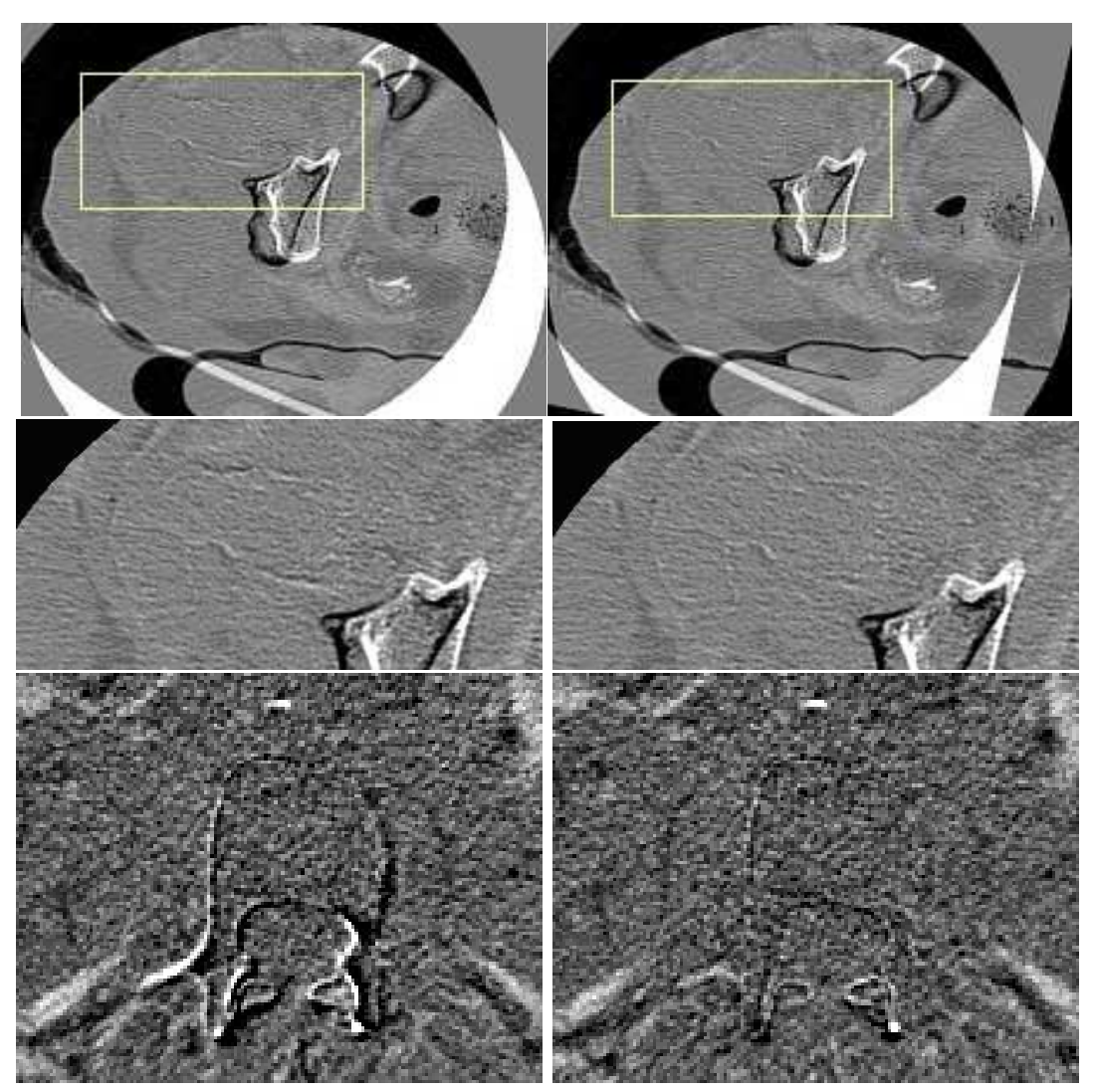

Fig. 3. CT-CT registration. Difference images (reference minus template), left: registration using grey value segmentation; right: improved registration with binary segmentation masks. Top: femur; center: femur regions of interest enlarged; bottom: spine. 
Fig. 4. Multimodal image registration for small animal imaging. Upper left: $\mu \mathrm{MR}$ dataset; upper right: $\mu \mathrm{CT}$ dataset. Bottom: blended MPRs (a reference with a registered template), left: registration using grey value 3D image stacks (reg1); right: improved registration when using binary segmentation mask for $\mu \mathrm{CT}$ (reg2).
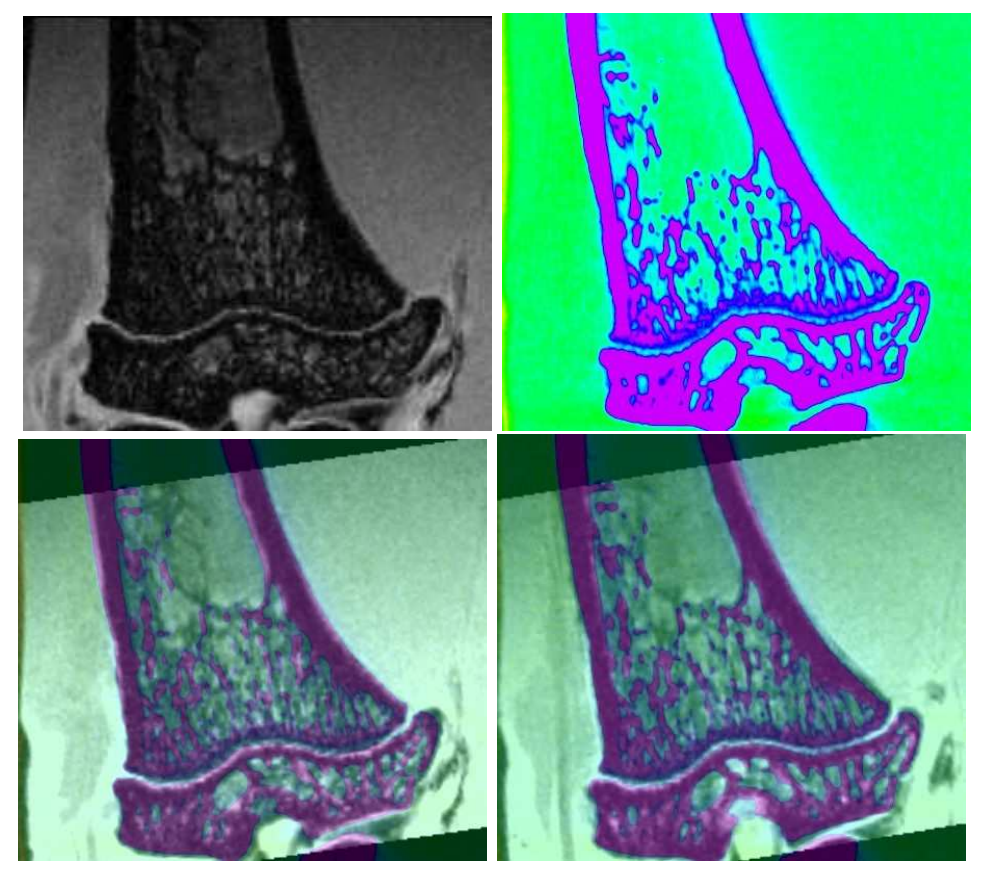

\section{References}

1. Hajnal JV, Hill DLG, Hawkes DJ, editors. Medical Image Registration. CRC; 2001.

2. Wang L, Greenspan M, Ellis R. Validation of bone segmentation and improved $3 \mathrm{D}$ registration using contour coherency in CT data. IEEE Trans Med Imaging. 2006;25(3):324-334.

3. Yezzi A, Zöllei L, Kapur T. A variational framework for integrating segmentation and registration through active contours. Med Image Anal. 2003;7:171-185.

4. Kang Y, Engelke K, Kalender WA. A new accurate and precise 3D segmentation method for skeletal structures in volumetric CT data. IEEE Trans Med Imaging. 2003;22(5):586-598.

5. Mastmeyer A, Engelke K, Fuchs C, et al. A hierarchical 3D segmentation method and the definition of the vertebral body coordinate system for QCT of the lumbar spine. Med Image Anal. 2006;10:560-577. 